\title{
AODV Protocol Improvement using Intelligent Clustering
}

\author{
Mohammed N. Abdullah \\ Assistant Professor, PhD \\ Dept. of Computer Engineering \\ University of Technology \\ Baghdad, Iraq
}

\author{
Mahmood Zaki Abdullah \\ Lecturer, $\mathrm{PhD}$ \\ Dept. of Computer Engineering \\ Al-Mustansiriyah University \\ Baghdad, Iraq
}

\author{
Rooa Adnan Sabri \\ Dept. of Computer Engineering \\ University of Technology \\ Baghdad, Iraq
}

\begin{abstract}
The paper proposes an algorithm for intelligent cluster head $(\mathrm{CH})$ election in clustered-AODV-based routing. Initially, the $\mathrm{K}$-mean for clustering the MANET nodes have been used according to their distances between them. Next, the intelligent $\mathrm{CH}$ election using soft computing techniques that includes sequential hybrid Fuzzy-Genetic controller for this decision making have also been applied. The paper simulation shows that the clustered-AODV-based routing protocol can be modified by changing the ordinary known AODV protocol (Classical AODV) to comply with the clustered network.
\end{abstract}

\section{Keywords}

MANET, AODV, Fuzzy-Genetic Controller, ClusteredAODV.

\section{INTRODUCTION}

The demand for self organizing, self healing networks without the interference of centralized or pre-established infrastructure/authority has been adopted to follow the increase in usage of wireless networks. Furthermore, soft computing techniques have shown a remarkable enhancement on network management. This has allowed network engineer to dedicate extra effort and time to the more specialized and complicated details of the system. The latter can be considered as challenged with decision-making based on partial information that performs adjustments on networks based on this partial information. However, such information can lead to difficulties and inaccuracies when automating network management using currently applied soft computing techniques [1]. One of promising soft computing techniques is the fuzzy reasoning. This technique will allow with help of information together with optimization algorithm such as Genetic algorithm to provide more flawless method of automating the network management decision making process [1].

In fact the flow of packets of information through the network needs to use some management rules and ways that ensure and increase the services provided over a network. In addition, network management assists the metrics, the cost, and operational cost under control. It is essential to mention that the management process of the network will adopt the capability of the delivery of packets through it that is because networking equipment is used effectively where it is needed the most. The conventional rules of managements uses simple ways of managing, like using extra equipment in the hardware design of network, while the newer ideas of managing employ intelligent aspects and protocols. The latter approach shows an increase the availability and quality of the services which the network provides. However, classical approaches may lead to disorder and disruption of networking services. This will be ineffective and gives poor utilization of feasibility [1].
Network management is the key to adapt and increase the investment of network that deliver large amount of packets of information without any disturbances and conflict [2].

It is important nowadays to manage the large size of network efficiently because the rise number of mobile nodes leads to many problems like extra cost of recourses, more number of hops, network congestion, and limited network lifetime which are very important factors in MANET. In addition it is important to use active routing protocol to reduce these problems.

There are various types of routing protocols in network community. The Ad hoc On-Demand Distance Vector (AODV) algorithm one of them, it enables dynamic, selfstarting, multi-hop routing between mobile nodes in ad hoc networks [3]. One of AODV routing protocol features comparing to other reactive routing protocol is the small size of packets in transmission [4].

The use of clustering solves many problems in MANET because of localized route discovery in Intra-Cluster Routing [4]. In Inter-Cluster Routing, the fuzzy-genetic can solve the head node selection problem by taking the effective factors of MANET and optimize the result to obtain the optimum head nodes in the network.

Since cluster heads must perform main work in each cluster, and it is important for routing and network reliability [5]. Thus care should be considered in cluster head election process. The latter should consider cluster head function, nodes with a higher degree of relative stability (less mobility), higher energy, and less distance between each other.

The proposed Cluster-AODV scheme uses clustering architecture and AODV functionalities to perform routing with intelligent selection of head nodes in the clustered network by using the input parameters of Fuzzy Inference system as input to the equation of the fitness in Genetic algorithm which is select according to distances between head nodes, mobility, and energy of the nodes in the network that is to say more than one important parameter in MANET.

The objectives of the research include important points: 
1. Examine the use of fuzzy reasoning in the area of routing aspect of configuration management. This leads to discover an effective method for routing data through a computer network. And assist to solve some challenges in MANET routing such as dynamic topology, limited transmission signal, energy constrained, etc. These are often measured in an imprecise or inaccurate manner, thus we suggest using natural method as fuzzy reasoning to employ it in an improved routing scheme.

2. Explore the use of GA to select the optimum head nodes in the network for forwarding the data from source to destination in effective way.

3. Integrate the FIS with GA to obtain fitness function that meet the requirements of MANET.

4. Employ the K-mean clustering algorithm to overcome some weak points in AODV routing protocol as scales to a large network size, and unnecessary flooding RREQ packet across all nodes in the network even in the case of near destination node (within the same cluster or the next cluster).

\section{CLUSTER BASED ROUTING IN MANET}

A successful method for dealing with and manage mobile ad hoc networks is by partitioning the network into clusters. In this way it allows better management of mobile ad hoc networks. Clustering is a method which divides the network into groups $[6,7]$.

Clustering in computer network is a division of the network into different virtual groups, based on rules in order to discriminate the nodes allocated to different sub-networks. Main goal is to achieve scalability in presence of large networks and high mobility. Cluster-based routing is a solution to address nodes heterogeneity, and to limit the amount of routing information that propagates inside the network. It increases the routes lifetime, thus decreasing the amount of routing control overhead [7].

Although the clustering techniques solve many problems in MANET but it still be hard to implement and select the optimum $\mathrm{CH}$ in each cluster. There are various routing protocols and Cluster based protocols have been proposed for MANET and a well-known among them are DSR, AODV [8].

\section{PROPOSED INTELLIGENT \\ CLUSTER-BASED AODV}

\subsection{Cluster Management}

The clustering techniques present network scalability and lead to more efficient use of network resources. It can be used for resource management, routing and location management in order to reduce communications, unnecessary flooding RREQ packets and computational overhead. In this section, cluster structure, intelligent cluster head election and communication mechanisms have been presented.

At the beginning of the implementation and before the movement of the nodes (when time equal to zero), the setup phase needs to be initialized. In this state, each node in the network is assigned with specific information as follows:

- The current geographical location within the specified area.

- The battery power (0-1.4)

- The transmission speed node (mobility).

- The speed limit 0-10 m/sec with pause time $20 \mathrm{sec}$.

- The next geographical location after movement.
- Random way point mobility mode has been used by specifying the current and next position randomly. With presentation that each node move from its current position in straight direction to the next position in the specified speed.

- After assigning the prosperities of the network and the nodes in setup phase, the distance matrix is calculated to define the connectivity of the nodes and the distances between them. This process requires the use of the Euclidean equation based on the position coordinate of each node with its neighbors.

The choice of clustering algorithm is very important and affects the clusters' reliability. In the proposed modification, the network is divided into several multi-hop clusters using Kmean clustering algorithm. The K-mean clustering algorithm flowchart that has been adopted in design is shown in Figure (1). In the design, the K-mean has been implemented by clustering method different network sizes (20-100) nodes into various numbers of clusters (2-12) clusters. With most clustering techniques, nodes are chosen to act different roles according to a certain criteria. In general, there are three types of nodes ordinary nodes, gateway nodes, and cluster heads $(\mathrm{CH}) \mathrm{s}[9,10]$.

The main tasks of a cluster head is to calculate the routes for long-distance messages and responsible for inter-cluster communication and stores routing and cluster information $[4$, $10]$. This role is changing to other ordinary nodes in the case of incompetence. After a period of time the election of $\mathrm{CH}$ is repeated to ensure that new $\mathrm{CH}$ is the optimum one. In this case the second election does not require extra overhead because the current $\mathrm{CH}$ informs the rest of the members of the new $\mathrm{CH}$. Furthermore, every $\mathrm{CH}$ should preserve two extra tables, an intra-cluster node table and a k-cluster head table. The former table includes the IDs of all nodes within a cluster whereas the s k-cluster head table should have the IDs of the $\mathrm{CHs}$ of all other clusters. Bering in mind that $\mathrm{CHs}$ coordinate operation in each cluster and store shared information. The sharing achieves by periodically every node broadcasts a hello message to maintain information about its neighbors [4].

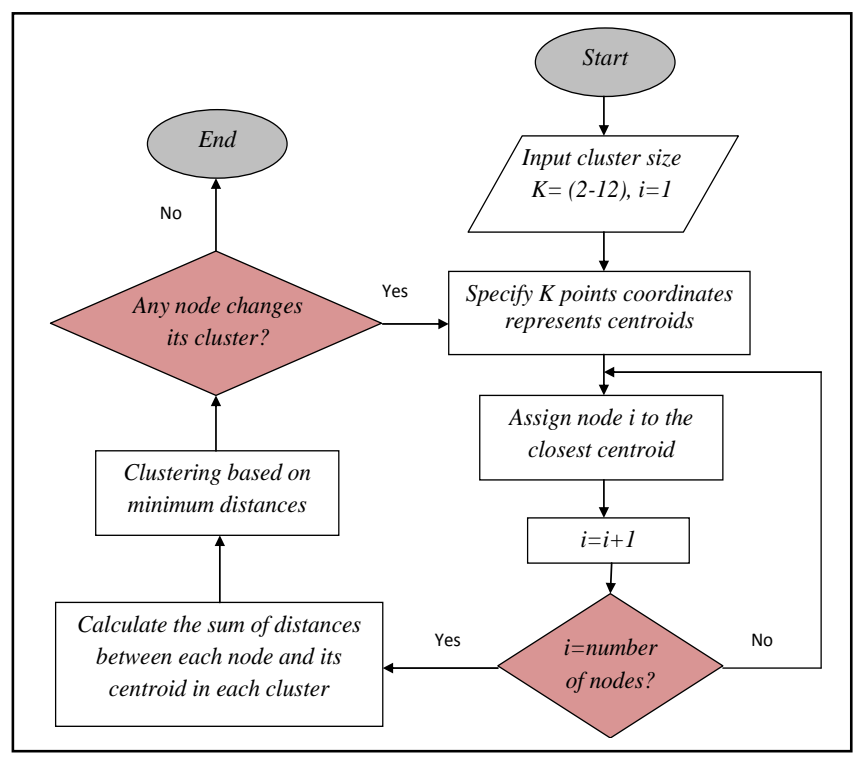

Fig 1: The K-mean clustering algorithm

Despite all the benefits of AODV protocol, there is a drawback of network management complexity in the case of large network size. When the network's size increases, unnecessary RREQ packets flooding will increase. This 
problem can be solved by using clustering techniques to allow scalability, and make the network more manageable.

To implement the routing in clustered AODV network some modifications in the classical AODV protocol should be made. This is because it depends on the node's ID number to discover the route by flood RREQ packets all over the network. This is in turn leads to resource exhaustion, and sometimes is not necessarily the shortest route path as shown in Figure (2).

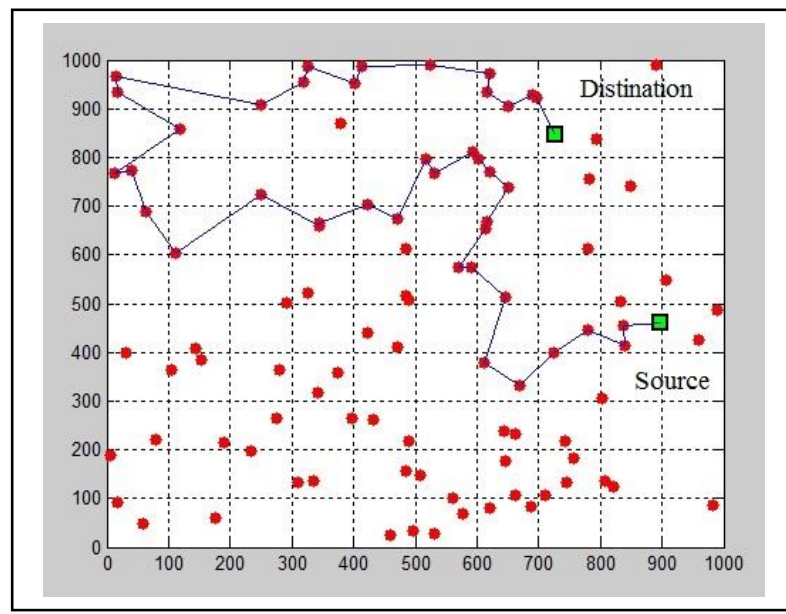

Fig 2: AODV Route Discovery for 100 nodes network

\subsection{Intelligent Cluster Head Election}

The challenge issue in the design is the intelligent head nodes election. This stage uses soft computing techniques (Fuzzy system and Genetic algorithm) to select the optimum head nodes that are able to forward the data through network in reliable way. There are various algorithms presented for cluster head election in network community. The proposed intelligent cluster head election design should pass through two stages: the genetic algorithm, and the fuzzy system. This assumption is made to ensure that the integration of these two tools will help to get the beneficial of both techniques.

\subsubsection{Genetic Algorithm Stage}

In this stage, the proposed process of GA when applied to optimize the $\mathrm{CH}$ election has been described. Table (1) shows the control parameters of GA maintained in the simulation.

Table 1. The control parameters of GA and its values

\begin{tabular}{|c|c|}
\hline Control Parameter & Values \\
\hline Population size & 20 \\
\hline Max. Generations & 100 \\
\hline Chromosome length & Cluster Size \\
\hline Crossover type & One point crossover \\
\hline Mutation rate & 1/Cluster Size \\
\hline
\end{tabular}

The GA stage involves the following steps;

- Chromosome Encoding step: The first step in GA design is encoding the chromosomes. In the adopted design, this will be the $\mathrm{CHs}$ of the network. Therefore the chromosome length is variable as the cluster size.
For example when the cluster size is five the number of genes in one chromosome is five and each gene represent the ID of each $\mathrm{CH}$ in the network as shown in Figure (3). For the network size 100 node, the ID should not exceed 100. In the simulation, different cluster size needs to be investigated from two clusters to twelve to see the impact of varying cluster size to the performance criteria. This means that the chromosome length should vary from two to twelve.

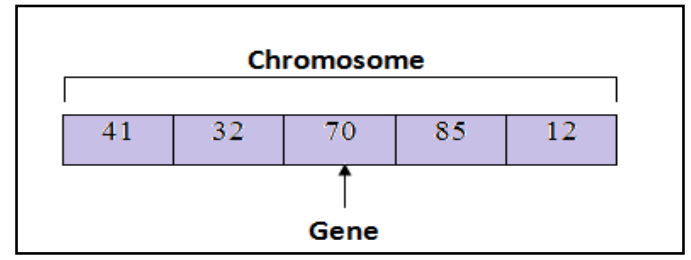

Fig 3: Chromosome representation when cluster size is five and network size is $\mathbf{1 0 0}$ nodes.

- Initial Population Generation step: The initial population generation is selected randomly from the set of chromosomes on the condition of select one node from each cluster as a candidate $\mathrm{CH}$ in that cluster. When the initial population generation selection is near the optimum the process will be faster to reach optimization in short time.

- Fitness Evaluation step: The fitness evaluation is the important step in the algorithm because it decides the accuracy of the optimization algorithm. It is determine which one of the population is the best to survive to the next generation and become strong candidate to win the optimization.

The proposed algorithm uses a combination effecting factors that represent weight metric to search dominant set. The combined weight is composed by three effecting factors. These are;

i- Battery power of $\mathrm{CHs}(\mathrm{E})$

ii- Distances between CHs (D)

iii- Mobility of CHs (M)

All these factors can be combined together in one equation that represents the fitness equation.

$W$ out $=\frac{w 1 \times E}{w 2 \times D+w 3 \times M}$

Where w1, w2, w3 are the weighting factors for the corresponding system control parameters and Wout is the fitness output. Each one of these effecting factors can be obtained from the node information and behavior. The weighting factors will be treated in FIS stage.

- Crossover and Mutation step: In the first operator, one point crossover has been used to generate new chromosomes from old ones. Next, the population generation sorted based on the fitness evaluation from the best to the worst candidate's chromosomes and crossover between each pairs as shown in Figure (4). The mutation operation is very simple but important to prevent the locally optimal result. 


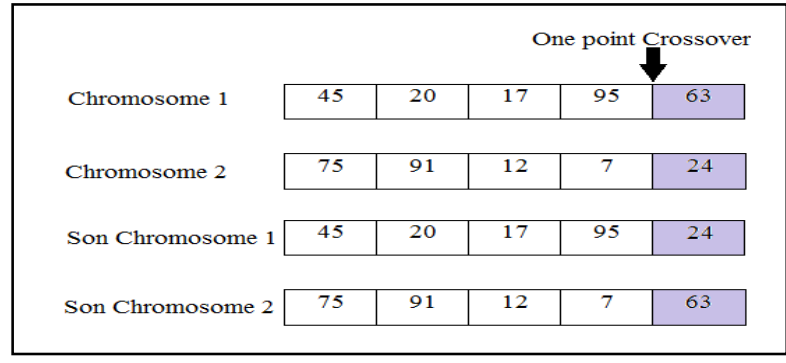

Fig 4: The one point crossover in five cluster size and 100 network size.

- Selection step: This step chooses which chromosome will be kept in the next generation and which one will be rejected. This process obtained by evaluating the best chromosomes in the population using the following equation:

\section{Fitness $[$ pop] $=\max (\mathrm{W}$ out $)$}

Where Fitness represents the vector of descending order from best to worse chromosomes after GA operation and pop represents the population size of GA. The best fitness of the population represents the best $\mathrm{CHs}$ elected according to all the three parameters described before.

Finally, the algorithm can be repeated several times to get the best results. This is because of the first attempt is not necessarily gives optimum chromosomes. This repetition in the algorithm called the iteration number which needs a stop condition to be deployed in the proposed system.

\subsubsection{Fuzzy Logic Controller Stage}

The main challenge in the optimization of $\mathrm{CH}$ election for MANET is to propose a reliable algorithm that can be prepared to the wide variety of circumstances in any MANET environment. The algorithm should react professionally in environments experience such as high nodes mobility, limited battery power and many wireless transmission constraints. Because it is often difficult to know which node will suffer from its environment and because the environment can change unpredictably, the algorithm must be able to adapt automatically. The Fuzzy Logic Controller (FLC) is a highly adaptive algorithm that can be used to achieve these aims.

The proposed algorithm includes the use of FLC in addition to the use of GA mentioned before. The main reason of this combination is to model and adapt GA control parameters seeking to improve the system performance. The FLC has been employed for dynamically computing suitable GA control parameters using the experience and knowledge of the GA experts. The adaptive change of the selected GA parameters is through Fuzzy Inference System (FIS) on the base GA feedback.

The FIS is a common computing structure based on the concept of fuzzy set theory, fuzzy if-then rules, and fuzzy reasoning. The FIS is used to control GA parameters which are used as weighting factors in the fitness equation. Initially the crisp input values should be determined, and then handle it in FIS model to produce crisp output that can obtained by using of defuzzification. The crisp inputs and output represents the weighting factors of GA fitness equation that mentioned before. Mamdani fuzzy inference engine has been used in the FIS editor model as shown in Figure (5).

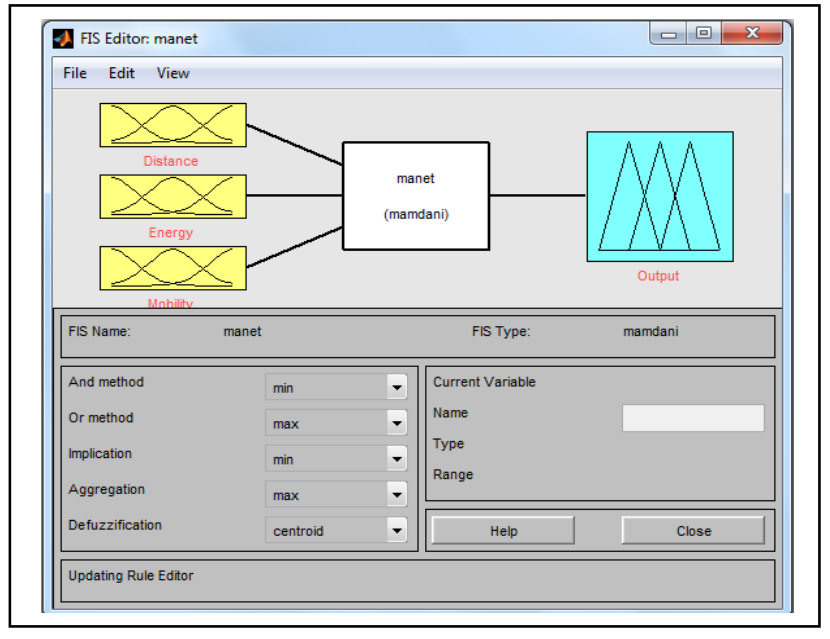

Fig 5: FIS Editor in MATLAB.

The important issues in the fuzzy rules are to keep the output weighting factor as maximum as possible in the following cases:

1. Minimum weighting factor of average distance between elected $\mathrm{CHs}$

2. Maximum weighting factor of average battery power (energy) of elected CHs.

3. Minimum weighting factor of average mobility of elected $\mathrm{CHs}$

The linguistic variables for the inputs and output membership function with the ranges of each can be shown in Table (2)

Table 2. The linguistic variables of inputs and output

\begin{tabular}{|c|c|c|c|c|c|c|c|}
\hline Parameter & $\begin{array}{c}\text { Input/ } \\
\text { output }\end{array}$ & Range & \multicolumn{4}{|c|}{ Linguistic Terms } \\
\hline Distance & Input & $0-1500$ & \multicolumn{2}{|c|}{ Near } & \multicolumn{2}{|c|}{ Median } & Far \\
\hline Energy & Input & $0-1.4$ & $\begin{array}{c}\text { Very } \\
\text { Low }\end{array}$ & Low & Median & High & $\begin{array}{c}\text { Very } \\
\text { High }\end{array}$ \\
\hline Mobility & Input & $0-10$ & $\begin{array}{c}\text { Very } \\
\text { Low }\end{array}$ & Low & Median & High & $\begin{array}{c}\text { Very } \\
\text { High }\end{array}$ \\
\hline Output & Output & $0-1$ & \multicolumn{1}{|c|}{ Weak } & \multicolumn{2}{|c|}{ Median } & Strong \\
\hline
\end{tabular}

On the hand the fuzzy operators that used in the proposed FIS model is: AND operator (Min), OR operator (Max), and Defuzzification operator (Centroid). These operators are used to join the fuzzy control rules of our system.

The FLC solves the problem of unknown weighting factors in the GA fitness equation which in turn leads to success in making decision of $\mathrm{CH}$ election without the need of administrator supervision. Figure (6) shows the overall proposed $\mathrm{CHs}$ election procedure. 


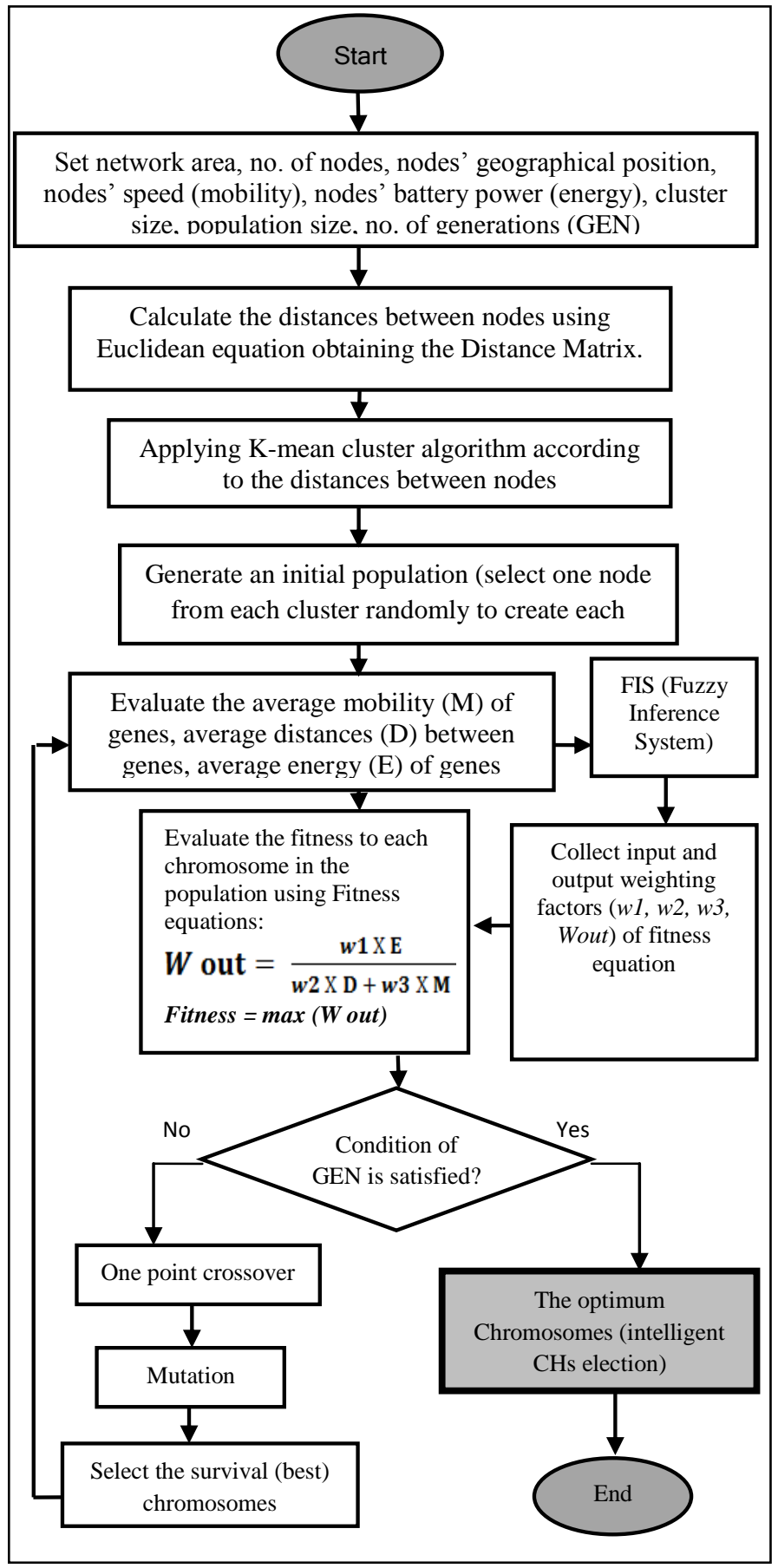

Fig 6: The proposed CHs election flowchart.

\subsection{Clustered-AODV-based Routing Stage:}

In this stage changes in AODV protocol has been implemented to be in line with intelligent clustering technique in previous stage. After clustering and elects $\mathrm{CHs}$ in intelligent way, two types of routing in the cluster-AODV network have been specified.

Intra-Cluster Routing phase: Since AODV is a reactive routing protocol, the source and destination nodes are specified first then the routing procedure will begin. If the source and destination located in one cluster only, the AODV routing algorithm will work within this cluster without flooding the RREQ packets outside for searching the route.
This is done by choosing the shortest path between the source and destination in ordinary way.

Inter-Cluster Routing phase (the intelligent phase): The best route between the $\mathrm{CHs}$ in the network is discovered which represents the optimum distance (minimum distance). This route will be considered as the backbone or the highway between the clusters to communicate each other directly through it. There are two situations in inter-cluster routing:

The first situation when the source and destination located in different clusters which are far to each other (out of transmission range of signal). The $\mathrm{CHs}$ of them cannot connect directly because of limited transmission range and the packet cannot be reached. In this case the source node uses classical AODV routing protocol inside its cluster to reach the $\mathrm{CH}$ by considering it as a temporary destination. And then connect to the $\mathrm{CH}$ of the destination through the backbone route. After reaching the destination $\mathrm{CH}$ return to use classical AODV routing protocol to reach the final destination in the cluster. This means that the route outside the cluster treated proactively not reactively until reach the $\mathrm{CH}$ of the destination node, then the packet forward to the destination node by using the classical AODV protocol.

The second situation when the source and destination located also in different clusters but in the same transmission range of signal. In this case the $\mathrm{CHs}$ of them will be connected directly without need of use the backbone path of CHs. Because the $\mathrm{CH}$ of destination is reachable transmission range. This leads to reduce the resource exhaustion especially the $\mathrm{CHs}$ of other clusters that not participating in the communication.

\section{PROPOSED SYSTEM SIMULATION AND EVALUATION}

The overall goal of this simulation study is to evaluate the performance of the proposed intelligent system election to choose the optimum $\mathrm{CHs}$ in professional way. And evaluate the performance of existing wireless routing protocol AODV which assigned in the research as (Classical AODV) and the proposed (Modified AODV) in various numbers of nodes and clusters. The nodes are placed randomly and move arbitrarily in Random Way Point model. The simulation has been performed using MATLAB 2012, a high performance language for technical computing. MATLAB features a family of add-on application specific solutions called toolboxes. Very important to most users of MATLAB, toolboxes allow learning and applying specialized technology [11]. Table (3) shows the simulation environment parameters used in the evaluation.

Table 3. The simulation parameters

\begin{tabular}{|c|c|}
\hline $\begin{array}{c}\text { Network } \\
\text { proprieties }\end{array}$ & Value \\
\hline Area & $1000 \mathrm{~m} \times 1000 \mathrm{~m}$ \\
\hline Nodes & $20,30,40,50,60,70,80,90,100$ \\
\hline Cluster size & $2,3,4,5,6,7,8,9,10,11,12$ \\
\hline Mobility model & Random Way Point \\
\hline Battery power & 0 up to 1.4 \\
\hline Maximum speed & $10 \mathrm{mps}$ \\
\hline Pause time & $20 \mathrm{sec}$ \\
\hline Traffic & CBR \\
\hline Packet size & 512 Bytes \\
\hline Nodes placement & Random \\
\hline Routing algorithm & OSPF algorithm \\
\hline Routing protocol & AODV \\
\hline
\end{tabular}




\subsection{Simulation Model One}

We consider a network of nodes placing randomly in various arrangements (one source and one destination) within a $1000 \mathrm{~m} \times 1000 \mathrm{~m}$ area. The performance of intelligent $\mathrm{CH}$ election is evaluated by varying the network size (number of nodes) ranges 20 to 100 and cluster size divides into two groups: low clustered network (Group 1) 2 to 6 cluster and high clustered network (Group 2) 7 to 12 cluster.

The proposed intelligent $\mathrm{CH}$ election system is tested against three parameters i.e. Average energy, mobility degree and average distance between $\mathrm{CHs}$ to evaluate the reliability and optimality of the control system.

Figures (7) and (8) show the average energy of the intelligent $\mathrm{CHs}$ election. By this parameter, the performance of the system and whether it chooses the best $\mathrm{CHs}$ or not are examined. It has been noticed that the average energy (battery power) of the $\mathrm{CHs}$ in the system with group 1 and 2 ranges 0.8 up to 1.3 when the network and cluster size are varied. The majority ranges over 1 , while the battery power of all nodes (ordinary and $\mathrm{CHs}$ ) ranges 0 to 1.4 . This means that the control system selects the high limit of energies.

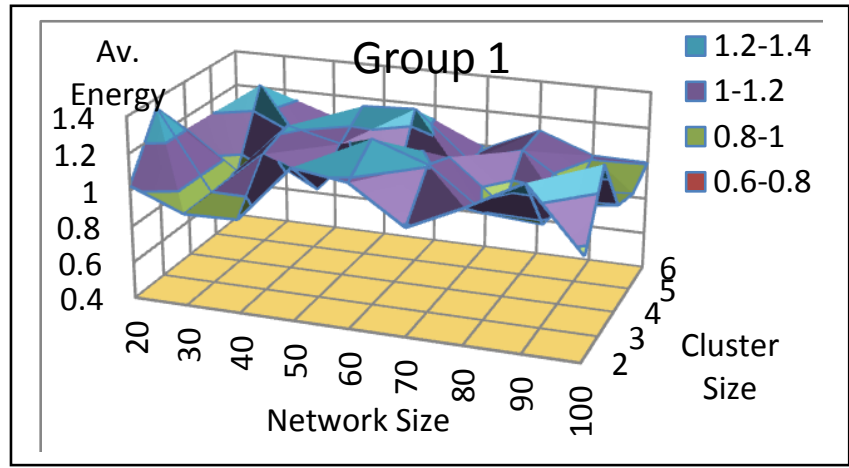

Fig 7: Average Energy of intelligent CHs election (Group1)

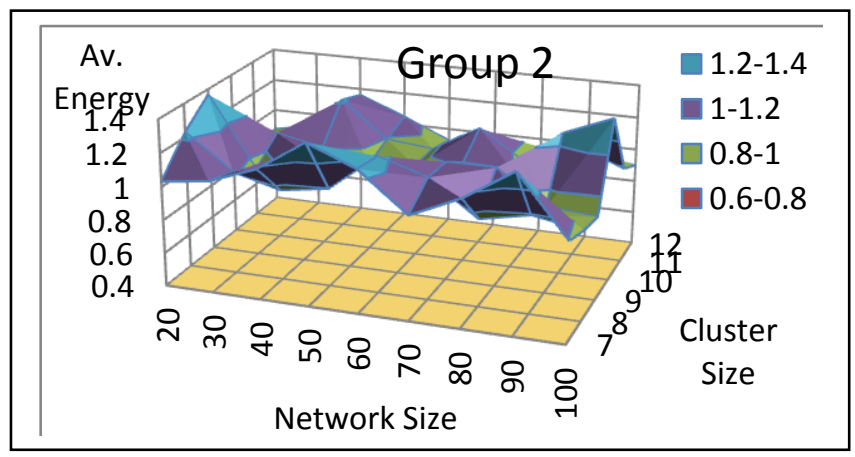

Fig 8: Average Energy of intelligent CHs election (Group2)

Figures (9) and (10) show the Mobility Degree of the intelligent $\mathrm{CHs}$ selection. By this parameter, the performance of the system and whether it chooses the best $\mathrm{CHs}$ or not are examined. It can be noticed that the Mobility degree (speed) of the $\mathrm{CHs}$ in the system in group 1 and 2 ranges 0 up to 7 when we varying the network and cluster size. The majority ranges from 2 to 4 , while the mobility of all nodes (ordinary and $\mathrm{CHs}$ ) ranges 0 to 10 . This means that the control system selects the lower limit of energies.

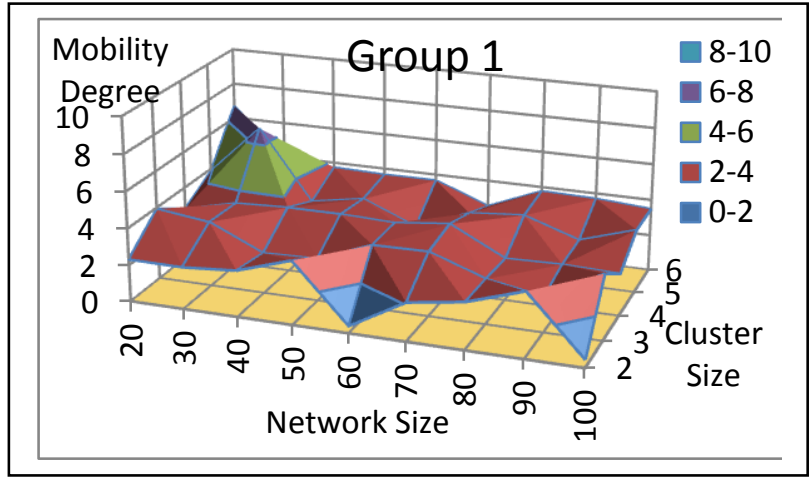

Fig 9: Mobility Degree of intelligent $\mathrm{CHs}$ election (Group1).

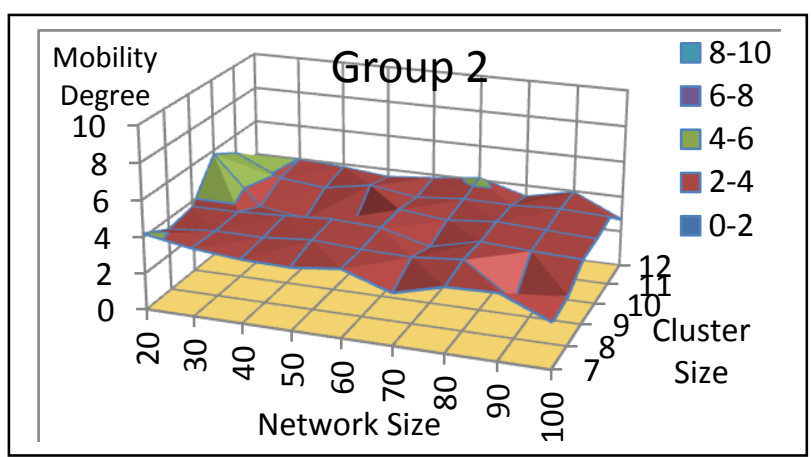

Fig 10: Mobility Degree of intelligent $\mathrm{CHs}$ election (Group2).

Figures (11) and (12) show the average distance of the intelligent $\mathrm{CHs}$ election. By this parameter, the performance of the system and whether it chooses the best $\mathrm{CHs}$ or not are evaluated. It has been shown that the average distances between $\mathrm{CHs}$ in the system in group 1 and 2 ranges 200 up to 700 when the network and cluster size are varied. It has been also shown that the majority in group 1 range 200 to 400 and group 2 ranges 400 to 600 which are within the transmission range of the system. This means that the control system selects the best CHs distribution.

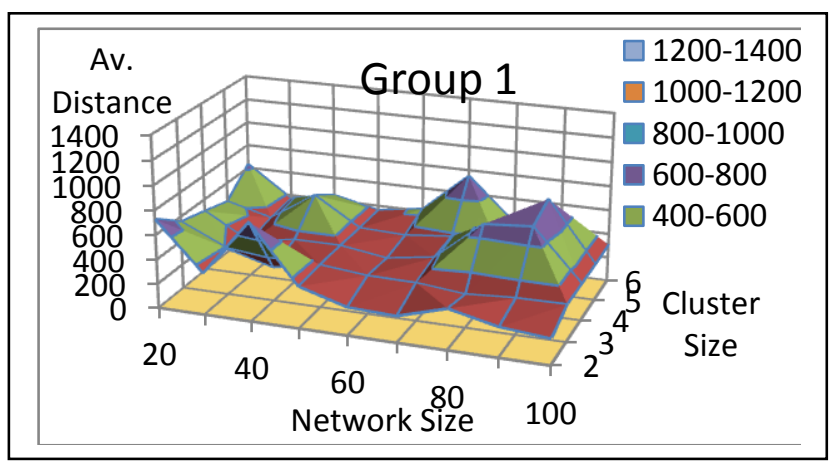

Fig 11: Average Distance of intelligent CHs election (Group1). 


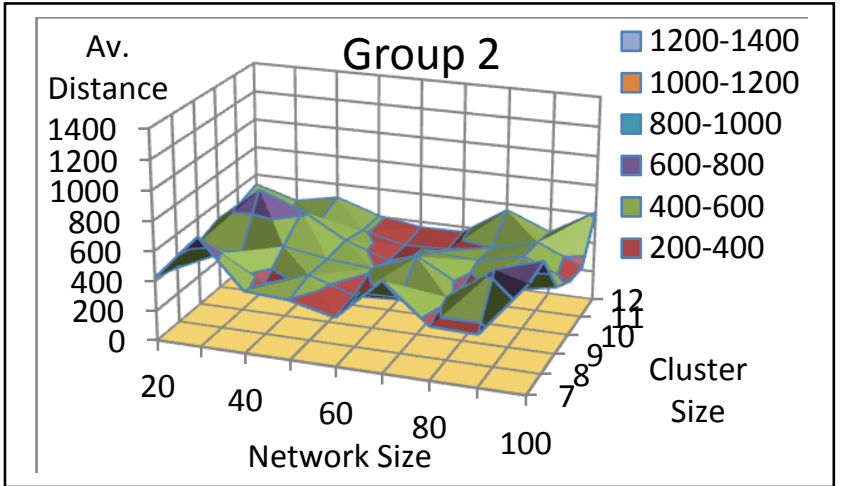

Fig 12: Average Distance of intelligent $\mathrm{CHs}$ election (Group2).

\subsection{Simulation Model Two}

Model two considers a network of nodes placing randomly in various arrangements (one source and one destination) within a $1000 \mathrm{~m}$ X1000m area. The performance of Classical AODV and Modified AODV is evaluated by keeping the cluster size (number of clusters) constant and varying the Network size (number of mobile nodes) ranges 20 to 100 in different cases of cluster ranges 2 to 12 .

Initially, the simulation has been conducted without applying the intelligent $\mathrm{CH}$ election (Classical AODV protocol). Next, the simulation is conducted by applying the proposed intelligent $\mathrm{CH}$ election and modified AODV routing in the same conditions to measure the performance of each situation and make the comparison between them. The proposed intelligent Modified AODV and the classical AODV are tested against three parameters i.e. NOH, Throughput, and PLR to evaluate the reliability and efficiency of the proposed system.

The simulation runs several times (12 cases) to ensure different cluster source and destination location (Inter-Cluster Routing). With in each case, the simulation measures the performance parameter. In all cases the MANET has been divided into 2 up to 12 clusters using K-mean clustering algorithm. With the use of proposed intelligent control system to elect the $\mathrm{CHs}$ the simulation has been executed to obtain the throughput, PLR and NOH results.

In case of 2 clusters, it has been found that the throughput of the modified AODV is better when the network size is increased. Further evaluation has been achieved via changing the cluster size to 3 and 4 . The results show that the throughput in modified AODV is better than that over classical AODV. However, in case of cluster size 5, the results show disparity in the throughput. When cluster size is 6 , the throughput has been increased in most network sizes. This is true until cluster size became 7 and 8 . In the latter cases, the throughput of modified AODV exceeds classical AODV after increasing the network size over 60 nodes. It can be noticed that when the clusters size is increase in the network better throughput can be achieved as shown in figure (13) when cluster size is 10.

Finally in the case when there are 12 clusters in the network and the network size more than 50 nodes, the modified AODV reached the best throughput in relation to the classical AODV. However, when the network size less than 50 nodes the throughput of the modified AODV became worse than that of the classical AODV.

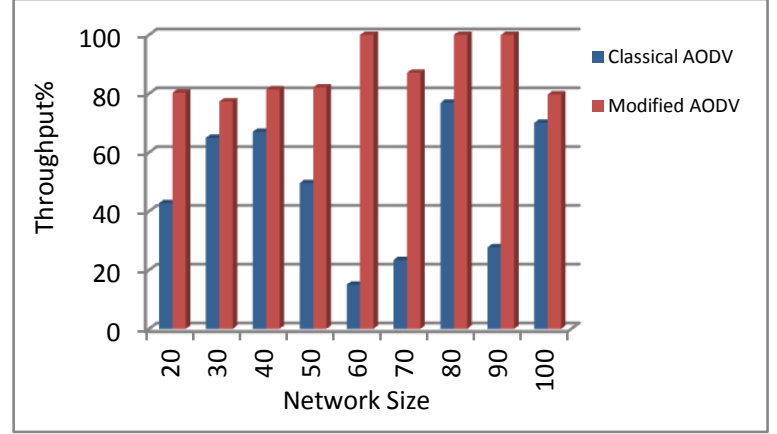

Fig 13: Throughput vs. network size when cluster size 10.

From the PLR aspects, the simulation results show that the modified AODV in cases 2, 3, 4, and 5 cluster sizes gives better performance when the network size is increased. This indicates that with the modified AODV the ratio of packet loss is decreased as the network size is increased especially with large number of clusters. This achievement continues to reach its best value when the cluster size becomes 6 . In this case the PLR record gives its best performance in almost all network sizes. This achievement PLR will not exceed 13.5\% in the worst case. However, the PLR with the cluster size 7, better performance of the proposed system can be achieved in the relation to classical one in all cases except the case of network size 30 nodes. Test with nodes over 60 has been conducted. The result shows that the PLR is decreased as number of nodes is increased. Test for PLR for the cases of 9 and 10 cluster size gives slightly better performance compared to classical AODV especially when the network size increased as shown in figure (14) when the cluster size is 10.

The advantages of the modified AODV compared to classical AODV ends when there are cases 11 and 12 clusters in the network. The results of PLR for the proposed system increased compared to the classical one and reach its worst value $29.6 \%$ when there are 11 clusters and $33.5 \%$ when there are 12 clusters in the network.

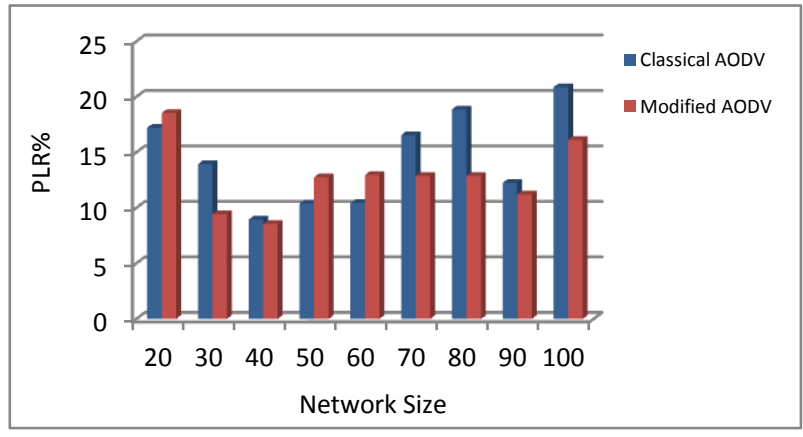

Fig 14: PLR vs. network size when cluster size 10.

Extra tests have been conducted to see the $\mathrm{NOH}$ required by modified AODV compared to classical AODV. It has been noticed that both systems give approximately the same performance for different network sizes in case of two clusters in the network. Test with other cases (cluster size 3, 4, 5... and 12) show that the proposed modified AODV gives better performance compared to classical AODV. Figure (15) shows the $\mathrm{NOH}$ when cluster size is 10 . 


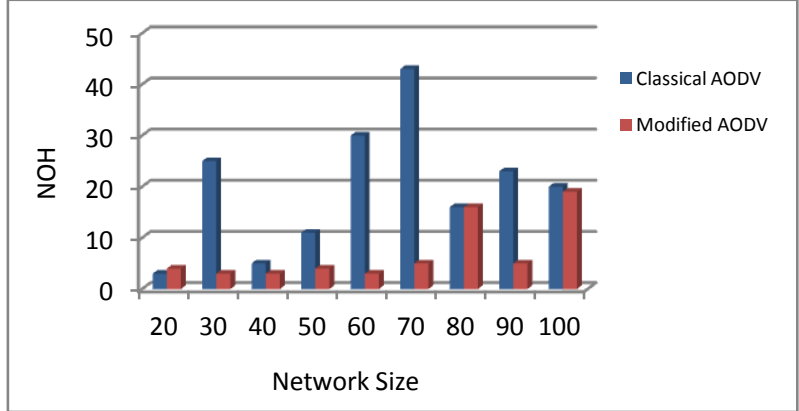

Fig 15: NOH vs. network size when cluster size 10.

\section{CONCLUSION}

The paper employs FIS and GA techniques in an attempt to improve cluster head selection in MANET. The results from the research give the following conclusions:

1. The proposed intelligent $(\mathrm{CH})$ election system shows high performance in various network and cluster conditions. This is because the proposed intelligent system consider all the affecting factors terms in MANET such as (distance, mobility, and energy) in the optimization that will lead to better network lifetime.

2. The $(\mathrm{CH})$ s election system is reliable due to fulfill the network requirements.

3. The use of FIS with GA presents an algorithm that convert the linguistic control factors based on administrator expert knowledge to search for optimum CHs.

4. The proposed system is more scalable because it shows better performance when the network size is increased.

\section{REFERENCES}

[1] Julia K. Brande, "Computer Network Routing with a Fuzzy Neural Network", PhD Thesis, Virginia Polytechnic Institute and State University, November 7, 1997.

[2] Alexander Clemm, "Network Management Fundamentals", Cisco Press, USA, 2007.
[3] C. Perkins, and S. Das," Ad hoc On-Demand Distance Vector (AODV) Routing", The Internet Society (2003).

[4] Mieso K. Denko, and Hua Lu, "AN AODV-Based Clustering and Routing Scheme for Mobile Ad Hoc Networks", IFIP International Federation for Information Processing, Volume 212, 2006, pp 83-97.

[5] M. Rezaee, and M. Yaghmaee, "Cluster based Routing Protocol for Mobile Ad Hoc Networks", February 23, 2009.

[6] B. An and S. Papavassiliou, "A Mobility-Based Clustering Approach to Support Mobility Management and Multicast Routing in Mobile Ad-hoc Wireless Networks," Int. J. Netw. Manag, vol. 11, no. 6, pp. 387395,2001

[7] A.E.Narayanan, R.Devi and Dr.A.Vincent Jayakumar, "An Energy Efficient Cluster Head Selection for Fault Tolerant Routing In MANET", International Journal of Engineering and Technology (IJET), Vol. 5, No. 2, AprMay 2013.

[8] N. Gupta, M. Shrivastava, and A. Singh, " Survey of Routing Scheme in MANET with Clustering Techniques", International Journal of Modern Engineering Research (IJMER), Vol. 2, Issue. 6, NovDec. 2012.

[9] Anju Sharma, Shini Agarwal, and Ravindra Singh Rathore, "Cluster Based Routing in Mobile Ad hoc Wireless Networks Using Neuro-Genetic Paradigm", International Journal of Scientific \& Engineering Research, Volume 3, Issue 7, July-2012, ISSN 22295518.

[10] Karamjeet Singh, "Energy Efficiency In Mobile Ad -hoc Networking Using Cluster Head Routing Protocol", International Journal of Advanced Research in IT and Engineering, Vol. 2, No. 5, May 2013.

[11] Website: WWW.MATHWORKS.COM the Mathworks Inc. 